NBER WORKING PAPER SERIES

\title{
ADVERSE SELECTION IN MEDICAID: EVIDENCE FROM DISCONTINUOUS PROGRAM RULES
}

\author{
Betsy Q. Cliff \\ Sarah Miller \\ Jeffrey T. Kullgren \\ John Z. Ayanian \\ Richard Hirth \\ Working Paper 28762 \\ http://www.nber.org/papers/w28762
NATIONAL BUREAU OF ECONOMIC RESEARCH
1050 Massachusetts Avenue
Cambridge, MA 02138
May 2021

The authors are grateful for assistance with data and project management from Erica Solway, Erin Beathard, Sarah Clark, and Lisa Cohn at the University of Michigan. We are also grateful to Michigan's Department of Health and Human Services for help with technical aspects of the program and support for the evaluation. Finally, we acknowledge valuable suggestions on our analysis from Jeffrey McCullough, Robert Lieberthal, Paul Jacobs, Tom Seldon and other participants in seminars at the Chicago Health Economics Workshop, American Society of Health Economists, and the Agency for Healthcare Research and Quality. The University of Michigan Institute for Healthcare Policy and Innovation (IHPI) is conducting the evaluation required by the Centers for Medicare and Medicaid Services (CMS) of the Healthy Michigan Plan (HMP) under contract with the Michigan Department of Health and Human Services (MDHHS). Data collected for this paper was funded by MDHHS and CMS for the purposes of the evaluation but does not represent the official views of either agency. DHHS had the right to review the paper for consistency with reports prepared by the authors under terms of the contract. The views expressed herein are those of the authors and do not necessarily reflect the views of the National Bureau of Economic Research.

NBER working papers are circulated for discussion and comment purposes. They have not been peer-reviewed or been subject to the review by the NBER Board of Directors that accompanies official NBER publications.

(C) 2021 by Betsy Q. Cliff, Sarah Miller, Jeffrey T. Kullgren, John Z. Ayanian, and Richard Hirth. All rights reserved. Short sections of text, not to exceed two paragraphs, may be quoted without explicit permission provided that full credit, including $\odot$ notice, is given to the source. 
Adverse Selection in Medicaid: Evidence from Discontinuous Program Rules Betsy Q. Cliff, Sarah Miller, Jeffrey T. Kullgren, John Z. Ayanian, and Richard Hirth NBER Working Paper No. 28762

May 2021

JEL No. I1,I12,I13

\section{ABSTRACT}

Recent expansions of Medicaid eligibility have come with increased experimentation with enrollee cost-sharing. In this paper, we exploit a discontinuous premium increase at the federal poverty level in Michigan's Medicaid expansion program to test low-income individuals' sensitivity to premiums using linked enrollment and claims data. At the cutoff, average premiums increase by $\$ 3.15$ and the probability of disenrollment increases by 2.3 percentage points. Increased disenrollment occurs among those with fewer documented medical needs at baseline, but not among those with greater medical needs. These results suggest healthier low-income individuals may be sensitive to even modest health insurance premiums, and that premiums may induce adverse selection in Medicaid plans.

Betsy Q. Cliff

University of Illinois Chicago

School of Public Health

bqcliff@uic.edu

Sarah Miller

Ross School of Business

University of Michigan

701 Tappan Street

Ann Arbor, MI 48109

and NBER

mille@umich.edu

Jeffrey T. Kullgren

University of Michigan

2800 Plymouth Rd., Bldg 16

Ann Arbor, MI 48103

jkullgre@med.umich.edu
John Z. Ayanian

University of Michigan

Institute for Healthcare Policy \& Innovation

ayanian@umich.edu

Richard Hirth

University of Michigan

rhirth@umich.edu

A data appendix is available at http://www.nber.org/data-appendix/w28762 
In the wake of Medicaid expansion under the Affordable Care Act (ACA), states have sought increased flexibility from the federal government in how to design and implement their Medicaid programs. Several states have implemented provisions that obligate Medicaid enrollees to pay for an increased share of their health care, either through utilization-based cost-sharing or regular monthly premiums. Others, particularly recent expansion states, are considering cost-sharing provisions as a way to link insurance expansion to conservative principles (Carroll 2018). Proponents of these provisions argue cost-sharing will promote personal responsibility among Medicaid enrollees and encourage them to make better decisions about their health care (Singer, Nelson, and Tipirneni 2017; Galewitz 2014). However, cost-sharing may impose a high burden on an already disadvantaged population, and may limit access to care by curtailing participation in Medicaid programs (Saloner, Sabik, and Sommers 2014). Prior studies have assessed whether premiums have meaningful effects on program participation in lowincome populations (Artiga, Ubri, and Zur 2017; Dague 2014; Finkelstein, Hendren, and Shepard 2019), but evidence is limited regarding effects in recent Medicaid expansion programs and on differences in price sensitivity by health status.

Michigan was one of the first states to pursue a federal waiver under the ACA to use increased cost-sharing in its Medicaid expansion program, including premium contributions (Ayanian 2013). Within the program, premium contributions of up to $2 \%$ of monthly income are required for enrollees with incomes at or above the federal poverty level (FPL). Co-payments are required for enrollees regardless of income for certain services. All costsharing responsibilities can be reduced through completion of a health risk assessment with a physician, and total cost-sharing is capped at $5 \%$ of income. ${ }^{1}$ The imposition of a premium creates a discontinuous change in costsharing at the FPL and levies the only cost-sharing responsibility unrelated to medical utilization. In addition, enrollees are not assessed program-related cost-sharing until after they have been enrolled for six months, providing a baseline period in which to assess differences in enrollee characteristics in the absence of cost-sharing responsibilities. We use the FPL cutoff in a regression discontinuity (RD) design to evaluate the impact of a monthly premium on enrollees' propensity to disenroll from the program, and use discontinuities in the average

\footnotetext{
${ }^{1}$ As a result, individuals with no income have no cost-sharing.
} 
amount of premiums to estimate the price sensitivity in this population. We further use enrollee health status in the baseline period to characterize which enrollees are more likely to disenroll.

Using administrative data on enrollment and enrollee characteristics, we find evidence that at the FPL cutoff disenrollment increases by 2.3 percentage points, a relative increase of $11.7 \%$. The size and significance of these effects, however, are somewhat sensitive to model specification, bandwidth choice, and method of inference. Because income relative to FPL is not perfectly measured and not all enrollees above the FPL are subject to premiums, we also implement a fuzzy RD approach to estimate the treatment effect of being exposed to premiums. We find that facing a premium increases disenrollment by 11.7 percentage points. For every $\$ 1$ increase in monthly premiums, we find an increase in disenrollment of 0.7 percentage points, which is similar to previous estimates of price sensitivity in low-income populations (Finkelstein, Hendren, and Shepard 2019), though slightly lower than estimates in other Medicaid populations (Dague 2014).

As premiums are not assessed until after six months of enrollment, we evaluate the heterogeneity of premium assessment across groups with different apparent baseline health care needs. Among those with either belowmedian health spending or no chronic disease claims in a baseline period that does not have cost-sharing, we find that facing a premium increases the likelihood of disenrollment by just above 13 percentage points. In these healthier subgroups, our disenrollment estimates are robust to a variety of model specifications and bandwidths. We estimate a $\$ 1$ increase in premiums is associated with a 0.8 percentage point increase in disenrollment in these two groups. By contrast, the effect of premiums on disenrollment among enrollees with above-median health spending or a chronic disease claim in the baseline period is smaller and statistically indistinguishable from 0 . We interpret our results as showing that Medicaid program participation for populations with more apparent clinical needs is not substantially affected by the imposition of premiums, but that healthier enrollees do respond to premium cost. These results align with recent work showing low-income households in the subsidized individual insurance exchange market with fewer expected medical costs are more price sensitive (Tebaldi 2017) .

Our study adds to the small but growing literature on how insurance premiums in Medicaid affect enrollment. Dague (2014) used a similar discontinuity to examine the effects of a premium on enrollment for adults in a 
slightly higher-income population. However, to our knowledge, no analysis has been able to characterize the health attributes of marginal Medicaid recipients who disenroll due to a premium or describe how premiums change the composition of Medicaid enrollees. Our results imply premium-based cost-sharing may induce adverse selection in these plans, by incenting healthier enrollees to leave at higher rates than they otherwise would. That finding has implications for the expected spending and risk-adjustment for the remaining Medicaid population. Additionally, our results have implications for other insurance markets that target low-income populations, such as the ACA health insurance exchanges. The high degree of sensitivity to even very small premiums documented here suggests that subsidies would need to cover nearly all premium costs if they are to induce healthy enrollees to remain in the market. That inducement may be even more crucial to maintain enrollment in the current environment, since the tax penalty related to the ACA's individual mandate was eliminated by Congress in 2017.

\section{Background and Related Literature}

Our study contributes to evidence of the impact of cost-sharing measures on the Medicaid population and lowincome individuals' price sensitivity to insurance costs more broadly. Prior to the ACA, states had less flexibility to implement cost-sharing for the Medicaid population, although some adopted premiums in their Children's Health Insurance Programs (CHIPs). Studies of those programs found a negative relationship between premiums and program participation (Buchmueller, Ham, and Shore-Sheppard 2015; Marton, Ketsche, and Zhou 2010; Kenney et al. 2006; Artiga, Ubri, and Zur 2017). Few studies have looked directly at the impact of premiums on enrollment in the adult Medicaid population. A study of Wisconsin's joint Medicaid and CHIP program, which imposed premiums for families beginning at $150 \%$ of the federal poverty level in 2008 , found that going from a $\$ 0$ to a $\$ 10$ monthly premium was associated with a 12 percentage point increase in probability of disenrollment within a year (Dague 2014). A second study found that enrollment in Oregon's Medicaid program declined by $44 \%$ within the first 6 months after implementation of monthly premiums for some members in 2003 (Wright et al. 2010). Most relevant to this context, premiums structured in a similar way as those in our study may have 
limited coverage gains when Indiana expanded its Medicaid program under the ACA (Freedman, Richardson, and Simon 2018).

More broadly, prior work has found that low-income individuals have a low willingness to pay for health insurance. A study using results from the Oregon Health Insurance Experiment estimated that the value of Medicaid to enrollees is less than half the government's cost of providing it, implying willingness to pay below medical costs (Finkelstein, Hendren, and Luttmer 2019). Relatedly, studies of the subsidized private health insurance markets for low-income individuals in Massachusetts and California found enrollees are willing to pay less, sometimes much less, than their expected medical costs (Tebaldi 2017; Finkelstein, Hendren, and Shepard 2019). These findings demonstrate that cost-sharing, even at modest amounts, reduces uptake of insurance and contrast with conventional models of health insurance, in which willingness to pay is above expected medical cost for a risk-averse consumer. Both previous work and our study question the applicability of that model to lowincome populations.

We also contribute to literature showing heterogeneity in price sensitivity across health status. In particular, we find that healthier enrollees are more price sensitive, and more likely to disenroll from Medicaid when facing a premium, than are enrollees with apparent medical needs. This finding is closely related to recent work by Tebaldi (2017) who shows younger households with lower expected medical costs are less willing to pay for insurance coverage on the California exchange. It is also consistent with a large body of theoretical and empirical work on adverse selection against various health insurance markets including the subsidized individual market and the Medicare supplemental coverage market (Arrow 1963; Geruso and Layton 2017; Bundorf and Simon 2006; LoSasso and Lurie 2009). Because of the limited use of premiums in Medicaid prior to the ACA, there has been little opportunity to study the effect of premiums in this population. A priori, it is uncertain how premiums will affect enrollees in this setting. Low-income populations have greater access to implicit insurance through charity care, care provided at no or low cost to them, which can decrease their valuation of formal insurance. These populations also have higher liquidity constraints than people with higher incomes, and they may be unable or unwilling to pay even small cost increases. However, cost-sharing amounts in Medicaid are typically much lower 
than in private insurance and capped at $5 \%$ of income in our study population. Additionally, enrollees in Michigan's program have the opportunity to reduce their cost-sharing obligations through healthy behaviors and, in this setting, cannot be disenrolled for non-payment. Nevertheless, we find the standard model of adverse selection holds in this population: as prices increase, those with the lowest expected medical costs drop coverage. Indeed, we find demographically-adjusted average medical spending in the first six months of the program is $40 \%$ less for those who later disenroll than for those who remain in the plan. Even among disenrollers, however, average medical spending substantially exceeds average cost-sharing; unadjusted monthly medical spending for this group is $\$ 166$ while enrollees pay an average of $\$ 4.50$ in premiums each month.

\section{Setting and Data}

\section{A. Setting: Healthy Michigan Plan}

In April 2014, Michigan expanded its Medicaid program by creating the Healthy Michigan Plan (HMP) with enhanced federal funding provided by the ACA. It was among the first states with both a Republican governor and Republican legislature to expand the program under the ACA and received a Section 1115 waiver from the federal government to include several provisions aimed at encouraging enrollees to take responsibility for their health and care costs (Ayanian 2013). A key feature was higher cost-sharing than had historically been used in Medicaid. All enrollees with reported income are required to make copayments for certain medical services. Enrollees with incomes between $100 \%$ and $138 \%$ of the federal poverty level are additionally required to pay premiums regardless of their use of health care services.

All cost-sharing obligations, premiums and copayments, are based on service use or income and are not billed until after six months of enrollment in a Medicaid managed care plan. All cost-sharing is assessed through quarterly statements, which include both service-based copayments and monthly premiums (see sample statement, Figure S1), and is collected by an independent vendor working with the state. Enrollees generally are expected to pay monthly (1/3 of the quarterly statement) although they can pay all at once. Premiums are linked to income 
and household size; program-defined premium estimates for relevant years from the state for households with up to three enrollees are shown in Table $\$ 1$ and range from $\$ 20-\$ 45$ per month. Copayments tend to be much smaller than premiums. Except for an elective inpatient hospital stay, copayments are between $\$ 1-\$ 3$ for those under 100\% FPL and \$2-\$8 for those above 100\% FPL (Table S2). Many health care services, including labs, imaging, preventive services, and those associated with a chronic disease visit are not subject to copayments. Thus, enrollees are not always responsible for utilization-based cost-sharing each quarter even if they do use services. In practice, the average cost-sharing invoice for individuals above the FPL conditional on a positive invoice is smaller than theoretical estimates; over the first 18 months of the program, the average monthly invoice for both copayments and premiums in this population was approximately $\$ 10$. The discrepancy between the theoretical and actual invoiced amounts is primarily due to the ability to reduce cost-sharing by completing a health risk assessment.

All enrollees have an opportunity to reduce their premiums by half through completion of a health risk assessment with a primary care physician in which the enrollee agrees to work toward a healthy behavior goal. Additionally, certain populations are exempt from all cost-sharing including those who become pregnant while enrolled in HMP, enrollees under age 21, enrollees receiving nursing home or hospice care, Native Americans and Alaskan Natives, and individuals who are eligible for Children's Special Health Care Services (CSHCS). If an enrollee fails to pay required cost-sharing, after a six-month grace period, state law directs the state health agency to pursue certain penalties or avenues for collection including taking the overdue amount from tax refunds or state lottery winnings. Non-compliant enrollees may also give up payment reductions earned through the health risk assessment. Enrollees cannot be disenrolled or prohibited from services due to failure to comply with payment requirements. They can voluntarily disenroll from the program at any time by contacting their caseworker, via phone or in person at a state health agency office. (Now there is also an online way to access the program, though it was not available during our study period.) Enrollees are also required to complete renewal paperwork and redetermination of eligibility after 12 months of continuous enrollment; someone who wished to disenroll could do so by failing to complete that process. After an individual has left the program for a period longer than 2 
months, their cost-sharing obligations reset such that they have another 6-month period without obligations upon re-enrollment.

\section{B. Data}

We use administrative data obtained from the Michigan Department of Health and Human Services (MDHHS) and provided as part of a required evaluation of HMP under its federal waiver from the Centers for Medicare and Medicaid Services. Our data include monthly program enrollment, demographics (age, gender, race, county of residence, percent of federal poverty level) measured at the time of enrollment, and cost-sharing statements for the state Medicaid expansion population. We have both premium and copayment amounts by quarter, the period for which payment obligations are assessed, for any enrollee who was sent a cost-sharing statement, regardless of whether they were obligated to pay anything. Additionally, while we do not have all individual medical claims, we have information on total spending and key medical utilization measures derived from the administrative claims file. We have data for the entire population who enrolled between April 2014 through March 2015. We followed enrollees through September 2016.

We include only enrollees who are in an HMP managed care plan for at least seven continuous months such that they were eligible to receive at least one cost-sharing statement. We exclude enrollees who we could identify as exempt from cost-sharing, including those in nursing home care, hospice care, or special-needs Medicaid populations and enrollees 21 and younger in 2014. We also exclude those age 62 and older in 2014, who may become Medicare eligible on the basis of age during our study period. As a final analytic step, we exclude enrollees with no income ever reported, which excludes about half of those otherwise eligible. In our RD design, they should not affect our estimates and we test whether they do in sensitivity analyses.

We measure likelihood of disenrollment over the six months subsequent to an enrollee's seventh continuous month in the program, which is when the first premium statement would be expected: Prob (disenrollment by month $13 \mid$ enrollment at month 7). We define disenrollment as any enrollee who leaves the Medicaid program 
entirely for more than 6 consecutive months. Enrollees continuously enrolled for seven months in a managed care plan who then leave and come back to any Medicaid program within six months are retained in the data set as an enrolled individual, as we assume this represents something other than disengagement from the program (e.g. delayed completion of paperwork to maintain enrollment). Enrollees who switch out of an HMP managed care plan to another Medicaid program (post 7-months enrollment in managed care) are also defined as remaining in Medicaid. To be at risk for disenrollment through our study period, which ends in September 2016, we require enrollees to be in an HMP managed care plan by August 2015. If an enrollee has multiple 13-month episodes in a managed care plan, we include the first 13-month episode.

We define a contribution or copayment obligation as any positive invoice amount of either type when it is assessed, regardless of the amount or whether the invoice was paid. Monthly copayment or contribution obligations are further defined as one-third of the average of a quarterly contribution or copayment invoice received by an enrollee. These invoices include reductions from completion of a health risk assessment but not past due amounts.

\section{Data Limitations}

While we are able to leverage very granular and complete data for Michigan's Medicaid program, these data have some limitations. We observe income based on the amount reported to and verified by the state, corrected to reflect the state's most current information. However, this amount may be different from the income level originally reported to the state. For example, suppose an enrollee reported an income at 40\% FPL from January through September, and faced cost-sharing based on that level. Then, in September, the enrollee reported to the state a new job that began in June, which gave the enrollee an income at 110\% FPL. The state would correct the FPL from June through September in the data file, though the invoices from those months would reflect the previous income information. The dataset did not have reliable information about whether FPL was corrected between the time when cost-sharing was assessed and when we observe FPL, causing some potential mismeasurement of income in our data. To mitigate this limitation, we test whether reported income roughly corresponds to predicted cost-sharing obligations (Figure 1; Table S3) and whether we see dynamic manipulation 
of income over time (Table S4). Additionally, we adjust for enrollment date - the month HMP managed care enrollment is first reported - in regression models.

Second, we are unable to observe some features of the population that lessen or eliminate cost-sharing responsibilities. For example, we do not have complete claims information or other data flags that allow us to exclude pregnant women, who are exempt from cost-sharing. Some enrollees have characteristics we would typically associate with cost-sharing, but we do not observe positive invoices. We are unable to determine why cost-sharing was not levied on these enrollees. These measurement errors are not a particular concern for our empirical approach, but they do indicate that a "fuzzy" regression discontinuity design—where the discontinuity is used as an instrument for our variable of interest, monthly premiums — is appropriate for our setting.

Lastly, there is a potential relationship between cost-sharing and diagnosis of medical needs. Given that enrollees can reduce their cost-sharing obligations through completion of a health risk assessment, which occur at primary care visits, enrollees who use health services may be more likely to have lower cost-sharing obligations. This effect changes the marginal Medicaid enrollee who is affected by the premium. As a result, our estimates may not generalize to settings where no such ability to reduce premiums exists. For this reason, we both adjust for receipt of a health risk assessment in regression models, and estimate the elasticities of premiums for each subgroup in addition to the effects of having any premium.

\section{Population characteristics}

Table 1 shows the characteristics of the HMP population $>0 \%$ FPL who were enrolled for at least 7 continuous months, split by whether they were still enrolled after 13 months $(n=195,495)$. Compared to those who are continuously enrolled for at least 13 months, individuals who disenroll are more likely to be male, younger, and non-black minorities. Disenrollers also have lower mean monthly spending on healthcare (\$166 vs \$297) and a lower likelihood of receiving a statement with a copayment.

Plotting demographics of the population by FPL shows few differences at 100\% of FPL (Figure S2; Table S5). Age of enrollees shows a sine pattern across the FPL distribution; the percent of enrollees who are white and 
having a later enrollment date are directly related to percent of FPL. Notably, the proportion of enrollees completing a health risk assessment, which can affect cost-sharing amount, is weakly related to income and shows no discontinuities at the FPL. In contrast to the demographic and health risk assessment, however, there are distinct changes in premium obligations at the FPL (Figure 1; Table S3). Specifically, for those with an average income between 99 and just below 100\% FPL, 22.8 percent are billed a premium; those with an average income between 100 and 101\% FPL 41.2 percent are billed a premium. The average premium amount also jumps at the FPL, from $\$ 3.05$ to $\$ 6.77$. By contrast, we see no jump in the percentage of the population assessed a copayment or, despite a program-defined difference, amount of copayments at this threshold (Table S3; Figure S3). Across the population, the average copayment is approximately $\$ 0.84$. This continuity in copayment amounts could be due to the program-defined amount of copayments being similar in absolute terms for most services (especially with a $50 \%$ discount for completing a health risk assessment) or to the fact that most common services, including imaging and lab studies, are copay-exempt. Other analyses of this program found $80 \%$ of enrollees used at least one copay-exempt service; $56 \%$ used at least one copay-likely service without differences by FPL (Hirth et al. 2018).

Compared with those below 100\% FPL, a higher percentage of enrollees above the FPL disenroll from the program between 7 and 13 months (17.9 vs 24.6\%; Table S3). Indeed, a naïve regression of disenrollment on FPL returns a positive estimate. However, this estimate is misleading as a causal effect due to higher enrollment churn among those with higher incomes, particularly close to the eligibility cap at $138 \%$ FPL.

The correlation between cost-sharing obligations and likelihood of disenrollment differs depending on both whether the entire invoice is considered, or whether premium and copayments are separated. When considering the entire invoice, the probability of disenrollment by cost-sharing level differs depending on whether a person is above or below the FPL. For those below the FPL, for whom cost-sharing is almost entirely due to service-based copayments, higher cost-sharing is correlated with lower demographically-adjusted probability of disenrollment (Figure S4). However, as the figure shows, for those above the FPL, the relationship between disenrollment and cost-sharing is concave, peaking at approximately $\$ 75$ in 6-month costs and declining at higher amounts. When 
copayments are separated out, as in Figure S5, there is no difference by FPL; higher copayments are related to a lower subsequent probability of disenrollment regardless of income. These graphs suggest that premiums as assessed by HMP may impact enrollment decisions in a different way than do copayments for services. To test that hypothesis directly, we use a regression discontinuity analysis described below.

\section{Analysis: Regression Discontinuity Design}

Our analysis makes use of a policy that changes discontinuously at the FPL. Individuals whose household income falls just below this cutoff are less likely to experience premiums relative to those with incomes just above, even though they are likely similar across other dimensions. Although the probability of having a premium obligation increases discontinuously at $100 \%$ of the FPL, it does not jump from 0 to 1 (Figure 1); this motivates our fuzzy $\mathrm{RD}$ analysis in this setting. We therefore present two versions of the model. The first, a reduced form or sharp RD estimate, measures the discontinuous change in disenrollment propensity at the cutoff using the following functional form:

$$
\begin{gathered}
Y_{i}=\propto+\gamma_{1}\left(F P L_{i}\right)+\gamma_{2}\left(F P L_{i}\right) D_{i}+\beta D_{i}+\delta Z_{i}+e_{i} ;-h \leq X \leq+h \\
\text { where } D_{i}=1\left\{x_{i}>\bar{x}, \bar{x}=100\right\}
\end{gathered}
$$

In this equation, $Y_{i}$ is the probability that an enrollee who is in the program at 7 months is still enrolled in HMP at 13 months, as defined above. We are primarily interested in whether the effect changes discontinuously at the cutoff point, D, which is at $100 \%$ of the FPL. We adjust for individual-level demographic and program-related covariates (age in bands, gender, race, region of residence, receipt of healthy behavior reward, and enrollment date) captured in the vector $\boldsymbol{Z}_{\boldsymbol{i}}$. We estimate the discontinuity using a local linear regression with a mean squared error-optimal bandwidth to minimize bias. To facilitate comparisons across subgroups, we also show the results using other selected bandwidths in our main results. We use a triangular kernel for all specifications and conventional standard error approaches due to the discrete nature of the running variable (Kolesár and Rothe 2018). 
The above estimation measures the change in disenrollment probability in the entire sample around the cutoff, even though some of the individuals above the cutoff are not "treated" (i.e., charged a premium). We thus estimate a second version that scales this reduced form estimate by the change in the treatment probability at the cutoff to estimate the effect of the premium among those actually charged a premium using a fuzzy RD approach. From this estimate, we report effects of treatment on the treated reflecting the change in probability of disenrolling for enrollees with premium obligations.

In order for our approach to be plausibly causal, we assume that those with incomes just below the FPL and just above it are otherwise similar. For this assumption to be reasonable, it must be the case that enrollees are not able to precisely manipulate the characteristics that determine whether or not they are subject to the treatment, in this case, income relative to FPL (Lee and Lemieux 2010). While income is reported by enrollees, it is verified using paychecks, tax forms or other documentation by the state. This verification, along with the complication that the threshold value depends on both household size and income, may make it difficult for enrollees to precisely manipulate income around the threshold value.

We test whether percent of FPL is manipulated by looking cross-sectionally at the density of FPL and dynamically by examining whether enrollees over the threshold adjust their incomes to be below the FPL threshold to avoid paying the premium. We conduct these tests in several ways. Figure S6 shows a histogram of percent of FPL for our eligible population with income between $70 \%$ and 130\% FPL. This plot shows a small spike just above 100\% FPL and a larger spike near the eligibility cut-off of 133\%, but no apparent spike just below 100\% FPL, which is where we would expect bunching if FPL were being manipulated to avoid premiums. This visual spike is confirmed by a McCrary density test (Figure S7), which shows a log increase in density of 0.14 ( $\mathrm{p}=0.02$ ) just above 100\% FPL (McCrary 2008). We account for the potential effect of such bunching in sensitivity analyses by estimating donut hole regressions in which we leave out particular bandwidths of FPL (Barreca, Lindo, and Waddell 2016). We also test for the dynamic manipulation of percent of FPL that is differential lowering of income level by those with premiums, perhaps to avoid these payments. To test for this type of manipulation, we use a Markov transition matrix to look at probabilities of moving above or below the 
FPL between initial and final, or 13th, month of enrollment in a managed care plan. We find that $87.8 \%$ of those who initially report incomes over the $100 \%$ of FPL are still above that threshold in their final observation (Table S4). This likelihood does not differ substantially by whether a person is required to make a premium contribution or not, and aligns with other evidence from this population showing a lack of strategic manipulation of income in the context of Medicaid recertification (Pei 2017). Taken together, the evidence does not suggest that individuals report lower incomes either initially or over time to avoid paying premiums.

As previously discussed, we also examine whether demographic characteristics--age, gender, race, region of residence, healthy behavior reward receipt and enrollment date--vary discontinuously at the cutoff. Here, we do detect some small but statistically significant differences at the cutoff income for age, gender, and enrollment date, though not for race, region or receipt of a healthy behavior reward (Figure S2; Table S5). To account for these differences at the cutoff, we control for age, gender, race, region, enrollment date and healthy behavior reward receipt in all of our specifications unless otherwise noted.

\section{Results}

Figure 1 displays the relationship between the fraction of enrollees required to pay a premium and enrollee income relative to the FPL. There is an obvious discontinuous jump in this fraction that occurs at the threshold of $100 \%$ FPL. Figure 2 shows a graph of disenrollment plotted against FPL, with linear fit lines on either side of the discontinuity. There is some noise in the data, yet disenrollment rates do appear to be elevated for those with incomes above the $100 \%$ FPL threshold.

Table 2 presents the main results of our RD model; we discuss results at the MSE-optimal bandwidth and present other bandwidths for comparison. Column 1, which formalizes the relationship shown in Figure 1, shows that the estimated probability of a premium contribution increases by about 19 percentage points at the FPL cutoff. The mean monthly premium amount increases by $\$ 3.15$ at the cutoff (Column 2; see Figure S8 for the corresponding graph). In the third column, we show the change in the probability of disenrolling at the 100\% FPL cutoff, which 
corresponds to the sharp RD estimate. We find a statistically significant increase of 2.3 percentage points in the probability of disenrollment at the cutoff (standard error $=0.0085$ ); compared with the disenrollment rate of 19.5 just below the FPL, this estimate represents a relative increase of $11.7 \%$.

In the next two columns, we present the treatment effects of exposure to monthly premiums (column 4) and the amount of the premium (column 5). We find the assessment of a premium increases the probability of disenrollment by 11.7 percentage points (standard error $=0.045$ ). Each additional dollar of premium cost increases disenrollment probability by about 0.71 percentage points (standard error $=0.0028$ ). Both estimates are statistically significant at conventional levels $(\mathrm{p}<0.05)$. The premium elasticity estimate implies that with a price change of $\$ 3.72$, the average unadjusted difference in monthly premiums for those just above and just below the poverty line, an additional $2.6 \%$ percent of the population will disenroll.

\section{A. Subgroup analyses}

We next examine disenrollment stratified by health need, proxied by either higher than median medical spending or the presence of a chronic disease diagnosis in claims data, both measured during an enrollee's first continuous 7-month period of enrollment in which they are not obligated to pay copayments or premiums. (Invoices sent in the seventh month of managed care enrollment are due in the eighth month; our data show the modal invoice is received in the eighth month.) There are no differences in likelihood of belonging to one of these groups across the FPL (Figure S9). There is considerable overlap among the subgroups; approximately $75 \%$ of those with above median spending have a chronic disease diagnosis. As in the main analysis, these two subgroups have an obvious discontinuity at the FPL in both likelihood and amount of a premium (Figures S10 and S11). However, we estimate different effects of the premium on disenrollment for each group. Figure 3 plots the likelihood of disenrollment for each subgroup; the data and linear fit lines appear to show larger increases in disenrollment for those with lower medical spending or without a chronic disease diagnosis compared with groups with higher baseline medical needs. 
We present estimates from the regression discontinuity model described above on each subgroup in Table 3 and discuss the estimates at the MSE-optimal bandwidth. The reduced form estimates show that for those with either no chronic disease claims or below median medical spending, disenrollment jumps by 3.3 and 3.6 percentage points, respectively; for those with a chronic disease or above median medical spending the jump is smaller in magnitude and not statistically different from 0. In the fuzzy RD design, individuals across all subgroups have the expected strong first-stage coefficients (Table 3, column 1). The first-stage coefficients are higher for the groups with lower medical needs, possibly because those with higher median spending or a chronic disease diagnosis may be more likely to engage with the medical system through health risk assessment completion as discussed above, and thus lower their premiums. For those with below-median baseline expenses, having a premium increases their likelihood of disenrollment by 13.4 percentage points (standard error=0.587). For those with above-median baseline expenses, a premium obligation increases their likelihood of disenrollment by 4.5 percentage points, and this estimate is not statistically significant. For those without a chronic disease diagnosis, a premium contribution increases their likelihood of disenrollment by 13.6 percentage points (standard error $=$ 0.067); the estimate for those with chronic disease is negative (-3.1 percentage points) though the magnitude of the standard error (0.10) indicates the estimate is statistically indistinguishable from 0 . These results imply that premiums will induce adverse selection in the program; with adjustments via linear regression for demographic and program covariates, the mean medical cost of disenrollers in the first 7 months of program enrollment is \$175.84 compared with $\$ 293.15$ for those who stay enrolled (Table S7).

Price sensitivity also appears affected by clinical need. For every dollar increase in monthly premium for those with below-median spending, disenrollment increases by 0.82 percentage points (standard error=0.0036); for every dollar increase in monthly premium for those with above-median spending, disenrollment increases by a statistically insignificant 0.40 percentage points (standard error=0.0048). Similar patterns emerge when we model the difference in disenrollment stratified by whether an enrollee has a claim for a chronic disease (Table 3). An increase of $\$ 1$ in monthly premium increases the rate of disenrollment by 0.84 percentage points (standard error=0.0042) for those without a chronic disease claim within their first 7 months of enrollment, and by 
statistically insignificant 0.53 percentage points (standard error=0.0041) for those with a chronic disease claim. To compare price sensitivity of each group, we use the estimates with the same bandwidth (10). These estimates shows that the price sensitivity for enrollees with above-median medical spending or a chronic disease diagnosis is roughly one-third to one-fourth the magnitude of that for those without a chronic disease diagnosis or belowmedian spending. These estimates imply that for the average increase in premium of $\$ 3.72$, an additional $3 \%$ of apparently healthier enrollees will disenroll, while the same increase would cause less than 1 percent of more medically needy enrollees to leave the program.

\section{B. Sensitivity Analyses}

In Figures S12 and S13, we present the results from several alternative specifications and bandwidth selection procedures. First, we include testing sensitivity to a bandwidth selector with a small coverage error rate (CER) rather than optimizing the mean squared error (Calonico, Cattaneo, and Farrell 2016). This bandwidth selector optimizes the construction of the confidence interval and may be preferable if the focus is on the confidence interval rather than the point estimate itself. Second, we also estimate a global linear rather than a local linear model to ensure our results are not sensitive to the exact way in which we model the running variable. We also probe the robustness of our results to retaining enrollees with no income, following enrollees for 12 months (rather than 6) after their initial 7 months of continuous enrollment to ensure results are robust over a longer time period, and to using the minimum, rather than average, income observed for a household to construct the running variable, which allows to capture enrollees always above the FPL threshold. We additionally measure outcomes when disenrollment is defined as 2 months out of the program rather than 6 to explore whether disenrollment patterns changed at the HMP program defined point where cost-sharing reset, and taking the subset of enrollees who had no copayments to mitigate confounding by the potential for copayment increases at the FPL. Although most of these 13 additional specifications provide similar estimates to those reported in Table 2, using narrow bandwidths selected through the CER method we do find negative small impacts of the premium on disenrollment. We suspect this result is due to the bunching observed in Figure S6 and, indeed we find a 
statistically significant positive estimate using this same specification and dropping enrollees with $100 \%$ or $101 \%$ FPL incomes from the analysis.

Figures S14 and S15 show estimates of the reduced form effect for a series of placebo cutoff points from $20 \%$ FPL through 85\% FPL. In these tests, we are comparing the estimate at 100\% FPL to placebo cut-off points; evidence it is larger than most placebo cut-off points would provide reassurance the effect is not spurious. For the main effects we find that $46 \%$ of placebo cutoff points have an absolute value smaller than our main estimate. When we examined some of the larger estimates (some are more than $10 \%$ at the placebo cutoff point) they fell around eligibility cutoffs for other programs commonly used in this population, including the state's Temporary Assistance for Needy Families eligibility. It may be that enrollment in these programs keeps people attached to a greater degree to the Medicaid program, leading to an increase in disenrollment just beyond these thresholds. We know of no such programs with eligibility cutoffs at the FPL that would confound our estimates. Split by medical spending, we find that $60 \%$ of placebo values are smaller in absolute value than our estimated effect for those with low medical spending and $17 \%$ of values have an absolute value smaller than our estimated effect for the high medical spenders. Split by chronic disease diagnosis, $65 \%$ of placebo values are smaller in absolute value than our estimated effect for those without a chronic disease and $15 \%$ of our estimated values are smaller in absolute value than our estimated effect for those with a diagnosed chronic disease. These placebo cutoff results support an interpretation of the disenrollment results from the full sample as suggestive rather than conclusive and bolster the stratified results showing a relationship between clinical need and disenrollment. Finally, we performed donut-hole regressions in which we excluded symmetric bands of FPLs around the threshold value to test whether the estimates are sensitive to the specific behavior of observations around the cutoff and to potential FPL manipulation (Cattaneo, Idrobo, and Titiunik 2018; Barreca, Lindo, and Waddell 2016). Figures S16 and S17 show the results from those series of regressions for the entire sample and for each subgroup split by medical need. These estimates are clustered around the main estimates (shown with the red line in the figures) and the confidence intervals run through the main estimates in most cases. This analysis shows that dropping estimates 
just above the FPL—where Figures S6 shows a small amount of bunching — leads to higher estimates of the treatment effect; income manipulation in those bands may bias the main estimates downward slightly.

\section{Discussion}

We find a jump in disenrollment when monthly premiums are assessed in Michigan's Medicaid expansion program. That jump is evident among those with less medical spending or no chronic medical conditions, though not for those with chronic conditions or higher medical spending. These results indicate the imposition of monthly premiums may limit program participation, and that this effect is strongest in those who use less medical care, indicating that enrollees who remain in Medicaid programs that include premiums are less healthy on average than in Medicaid programs without premiums. While it may lower overall program spending, this result implies that the per-member spending in Medicaid programs with premiums is higher than in programs without premiums. Capitation based on membership should take into account the effect of cost-sharing on population health risk, particularly if initial risk adjustment fails to account for this disproportionate drop out of healthier enrollees in the Medicaid program.

Our results show lower disenrollment than earlier work on cost-sharing among Medicaid enrollees (Wright et al. 2010). Unlike Oregon, cost-sharing was a feature of the Healthy Michigan Plan from its inception, and some potential enrollees may have stayed out of the program entirely due to the prospect of cost-sharing. Also, Michigan's expansion both allowed enrollees to reduce their level of cost-sharing by completing a health risk assessment and remain in the program even if non-compliant with payment, though they could become subject to collections actions. Additionally, as noted above, enrollees who leave for more than 2 months and then return reset their cost-sharing schedule such that they are not subject to cost-sharing for 6 months after re-enrollment. These features may have limited attrition from the program and, thus, our results could represent a lower bound on price responsiveness. In surveys, the majority of HMP enrollees agreed the program is fair and affordable (Goold et al., 2018), and few who were no longer enrolled (2.1\%) reported dissatisfaction with costs as the 
primary reason for ending enrollment (Clark and Goold, 2018). Yet, even in these circumstances, in which enrollees can mitigate or even avoid cost-sharing altogether, premiums appear to encourage disenrollment among healthier enrollees.

Our elasticity results are similar to previous findings of high price sensitivity for insurance among low-income individuals. A closely related paper, Dague (2014), suggests that a \$1 increase in premium would cause a 1.2 percentage point drop in enrollment; our main estimate finds each \$1 in additional premiums causes a 0.7 percentage point drop. Given a churn of $20 \%$ just below the FPL threshold and an average increase of $\$ 3.72$ in premium amount at the FPL, our elasticity estimates demonstrate that in this program the imposition of premiums increases attrition by a relative 13 percent. Attrition from the program also occurs because of income increases, other health insurance coverage, or administrative problems with re-enrollment (Clark and Goold 2018). Previous work has shown that low income enrollees value insurance at less than its actuarial value (Finkelstein, Hendren, and Shepard 2019). We add to these findings by showing heterogeneity in insurance valuation by clinical need, consistent with the notion that premiums could contribute to adverse selection in this setting.

A clear policy implication from this analysis is that premium-based cost-sharing as implemented in Michigan may limit efforts toward higher rates of insurance coverage, as some people are unwilling or unable to pay the additional costs imposed through monthly premiums. We do not know the extent to which those who disenroll due to cost-sharing experience are subsequently uninsured. In a survey of former enrollees in Michigan's Medicaid program, $81 \%$ said they would have had no viable insurance options without the program, and 55\% said they had a gap in health coverage of longer than 3 months once they left the program (Clark and Goold 2018).

Because the premium amounts are lower than average expenses even for relatively healthy disenrollers, the shortrun effect of this policy would be expected to yield savings for public budgets, particularly the federal and state budgets for Medicaid expansion. In addition to a smaller Medicaid population, there are potential savings that arise from the fact that capitated payments to managed care plans are based on claims data from prior to the imposition of premiums, when the patient pool is relatively healthier. In Michigan, managed care plans are paid using capitated rates that are risk adjusted using encounter data for the case mix of each plan's population at the 
beginning of a fiscal year. ${ }^{2}$ As a result, in the short-run, the selection out of Medicaid by healthy enrollees

implies that the state both pays for fewer enrollees and underpays Medicaid managed care plans for the remaining patient population covered. However, those who disenroll may take advantage of implicit insurance (i.e. access to health care services outside of formal health insurance) offered by statute or safety-net health care providers, or of conditional coverage, the ability to enroll in Medicaid after a health shock (Cutler and Gruber 1996; Finkelstein, Hendren, and Luttmer 2019; Mahoney 2015). More research following those who disenroll from Medicaid due to premiums is needed to fully understand the experiences of these disenrollers and subsequent fiscal consequences. If those who disenroll continue to use medical services subsidized by the state or federal governments, the cost savings may be lower than expected or non-existent.

While states may save money by paying for remaining enrollees at a rate calculated on a healthier population, such changes could impact managed care plans' profitability and disrupt the managed care market. The revenue lost to managed care organizations in this setting when relatively healthy beneficiaries leave the program amounts to slightly less than $1 \%$ of their total revenue for the expansion population on average. ${ }^{3}$ These losses are not trivial; Michigan builds margins of about $2 \%$ into their capitation rates across plans (Tapperna et al. 2017). If losses are concentrated in a small number of plans or correlated with plan characteristics, they could be particularly disruptive. Previous work in Kentucky's Medicaid program has shown that higher-cost enrollees tend to switch plans more often, and move toward higher quality plans, than do low-cost enrollees (Marton, Yelowitz, and Talbert 2017). Like Kentucky, Michigan allows Medicaid enrollees to switch plans. HMP enrollees can

\footnotetext{
2 State of Michigan Contract No. Comprehensive Health Care Program for the Michigan Department of Health and Human Services (https://www.michigan.gov/documents/contract_7696_7.pdf) . Accessed March 30, 2021. Capitated plan payments are made from the state to plans based on monthly enrollment, fully re-calculated based on enrollee expected health risk annually and, to some extent, risk adjusted six months into the year to account for changes in enrollment (Brandel, Pettit, and Gordon 2018).

${ }^{3}$ We use a "back of the envelope" estimate to calculate the lost revenue as follows. Assume those who do not disenroll due to premiums have spending $\$ 272.67$ of while the 2.3 percent of enrollees who disenroll due to premiums have the average spending of the typical disenroller $(\$ 165.67$, Table 1$)$. Averaging these two groups results in the observed average expenditure we observe in the expansion population $(\$ 270.21$; Table S3). Assume further that plans receive as per member revenue average spending (\$270.21). After premiums are imposed, plans' revenue remains $\$ 270.21$ per member but costs per member become the average expenditure of those who do not disenroll only, $\$ 272.67$ per member. Loss is therefore $\$ 272.67-\$ 270.21=\$ 2.46$ per member per month, or about $0.9 \%$ relative to monthly revenue per member $(2.46 / 270.21)$.
} 
choose a plan at the beginning of their initial enrollment or annually during an open enrollment period. While we do not know whether the adverse selection we identify affects managed care plans differentially by quality, if lower-cost enrollees are more likely to leave health plans after premium imposition, and higher-cost enrollees both stay and gravitate toward higher-quality plans, it could result in higher-quality plans with a significantly costlier population over time. If not sufficiently compensated through regular risk adjustment, this effect may destabilize the Medicaid managed care market. Our results suggest this threat; future work is needed to measure and confirm the differential effect of cost-sharing across Medicaid managed care plans.

This study represents the Medicaid cost-sharing experience of one early-adopting Medicaid expansion state, which may limit its generalizability. Nonetheless, we believe our results show the potential for increased disenrollment in low-income populations with even modest premiums. States should be aware of this effect and of the potential for adverse selection when making policy decisions about enrollees' cost-sharing responsibilities. 


\section{References}

Arrow, Kenneth J. 1963. "Uncertainty and the Welfare Economics of Medical Care." The American Economic Review 53 (5): 941-73.

Artiga, Samantha, Peter Ubri, and Julia Zur. 2017. "The Effects of Premiums and Cost Sharing on Low-Income Populations: Updated Review of Research Findings.” Issue Brief. Henry J. Kaiser Family Foundation. https://www.kff.org/medicaid/issue-brief/the-effects-of-premiums-and-cost-sharing-on-low-incomepopulations-updated-review-of-research-findings/.

Ayanian, John Z. 2013. "Michigan's Approach to Medicaid Expansion and Reform." New England Journal of Medicine 369 (19): 1773-75.

Barreca, Alan I., Jason M. Lindo, and Glen R. Waddell. 2016. "Heaping-Induced Bias in RegressionDiscontinuity Designs.” Economic Inquiry 54 (1): 268-93.

Brandel, Shelley, Christopher Pettit, and Alisa Gordon. 2018. "University of Maryland, Baltimore County: Medicaid Managed Care Rate Setting and Payment Innovation Study." Milliman Inc. https://www.manatt.com/Manatt/media/Documents/Articles/Medicaid-Managed-Care-Rate-Setting-andPayment-Innovation-Study.pdf.

Buchmueller, Thomas, John C. Ham, and Lara D. Shore-Sheppard. 2015. "The Medicaid Program.” National Bureau of Economic Research.

Bundorf, M. Kate, and Kosali I. Simon. 2006. "The Effects of Rate Regulation on Demand for Supplemental Health Insurance." American Economic Review 96 (2): 67-71.

Calonico, Sebastian, Matias D. Cattaneo, and Max H. Farrell. 2016. Coverage Error Optimal Confidence Intervals for Regression Discontinuity Designs.

Carroll, Aaron. 2018. "Finally, Some Answers on the Effects of Medicaid Expansion." The New York Times, July 2, 2018, sec. The Upshot. https://www.nytimes.com/2018/07/02/upshot/finally-some-answers-on-theeffects-of-medicaid-expansion.html.

Cattaneo, Matias D., Nicolás Idrobo, and Rocío Titiunik. 2018. A Practical Introduction to Regression Discontinuity Designs: Volume I. Cambrigde University Press, forthcoming.

Clark, Sarah, and Susan Dorr Goold. 2018. "Report on the Healthy Michigan Voices 2016-17 Survey of Individuals No Longer Enrolled in the Healthy Michigan Plan." University of Michigan Institute for Healthcare Policy and Innovation.

Cutler, David M., and Jonathan Gruber. 1996. "Does Public Insurance Crowd out Private Insurance?” The Quarterly Journal of Economics 111 (2): 391-430.

Dague, Laura. 2014. "The Effect of Medicaid Premiums on Enrollment: A Regression Discontinuity Approach." Journal of Health Economics 37: 1-12.

Finkelstein, Amy, Nathaniel Hendren, and Erzo FP Luttmer. 2019. "The Value of Medicaid: Interpreting Results from the Oregon Health Insurance Experiment." Journal of Political Economy 127 (6): 2836-74.

Finkelstein, Amy, Nathaniel Hendren, and Mark Shepard. 2019. "Subsidizing Health Insurance for Low-Income Adults: Evidence from Massachusetts.” American Economic Review 109 (4): 1530-67.

Freedman, Seth, Lilliard Richardson, and Kosali I. Simon. 2018. "Learning from Waiver States: Coverage Effects under Indiana’s HIP Medicaid Expansion.” Health Affairs 37 (6): 936-43.

Galewitz, Phil. 2014. "Michigan To Reward Medicaid Enrollees Who Take 'Personal Responsibility." Kaiser Health News, June 11, 2014. 
Geruso, Michael, and Timothy J. Layton. 2017. "Selection in Health Insurance Markets and Its Policy Remedies." Journal of Economic Perspectives 31 (4): 23-50.

Hirth, Richard A., Elizabeth Q. Cliff, Jeffrey T. Kullgren, A. Mark Fendrick, Sarah Clark, Erin Beathard, Erica Soloway, Lisa Cohn, and John Z. Ayanian. 2018. "Report on the Impact of Cost Sharing in the Healthy Michigan Plan. Evaluation Domain V/VI." Submitted to the Michigan Department of Health and Human Services.

Kenney, Genevieve, R. Andrew Allison, Julia F. Costich, James Marton, and Joshua McFeeters. 2006. "Effects of Premium Increases on Enrollment in SCHIP: Findings from Three States." INQUIRY: The Journal of Health Care Organization, Provision, and Financing 43 (4): 378-92.

Kolesár, Michal, and Christoph Rothe. 2018. "Inference in Regression Discontinuity Designs with a Discrete Running Variable.” American Economic Review 108 (8): 2277-2304.

Lee, David S., and Thomas Lemieux. 2010. "Regression Discontinuity Designs in Economics." Journal of Economic Literature 48 (2): 281-355.

LoSasso, Anthony T., and Ithai Z. Lurie. 2009. "Community Rating and the Market for Private Non-Group Health Insurance.” Journal of Public Economics 93 (1-2): 264-79.

Mahoney, Neale. 2015. "Bankruptcy as Implicit Health Insurance." American Economic Review 105 (2): 710-46.

Marton, James, Patricia G. Ketsche, and Mei Zhou. 2010. "SCHIP Premiums, Enrollment, and Expenditures: A Two State, Competing Risk Analysis.” Health Economics 19 (7): 772-91.

Marton, James, Aaron Yelowitz, and Jeffery C. Talbert. 2017. "Medicaid Program Choice, Inertia and Adverse Selection." Journal of Health Economics 56: 292-316.

McCrary, Justin. 2008. "Manipulation of the Running Variable in the Regression Discontinuity Design: A Density Test." Journal of Econometrics 142 (2): 698-714.

Pei, Zhuan. 2017. "Eligibility Recertification and Dynamic Opt-in Incentives in Income-Tested Social Programs: Evidence from Medicaid/CHIP.” American Economic Journal: Economic Policy 9 (1): 241-76.

Saloner, Brendan, Lindsay Sabik, and Benjamin D. Sommers. 2014. "Pinching the Poor? Medicaid Cost Sharing under the ACA." New England Journal of Medicine 370 (13): 1177-80.

Singer, Phillip M., Daniel B. Nelson, and Renuka Tipirneni. 2017. "Consumer-Directed Health Care for Medicaid Patients: Past and Future Reforms." American Journal of Public Health 107 (10): 1592-94.

Tapperna, Sara, Jeff Goldman, Luke Smith, and Steve Tutewohl. 2017. "Medicaid Managed Care Organizations: Considerations for Calculating Margin in Rate Setting." Society of Actuaries. https://www.soa.org/globalassets/assets/Files/Research/medicaid-managed-report.pdf.

Tebaldi, Pietro. 2017. "Estimating Equilibrium in Health Insurance Exchanges: Price Competition and Subsidy Design under the Aca." Working paper. Becker Friedman Institute for Research in Economics Working Paper No. 2017-05. https://papers.ssrn.com/sol3/papers.cfm?abstract_id=3020103.

Wright, Bill J., Matthew J. Carlson, Heidi Allen, Alyssa L. Holmgren, and D. Leif Rustvold. 2010. "Raising Premiums and Other Costs for Oregon Health Plan Enrollees Drove Many to Drop Out." Health Affairs 29 (12): 2311-16. 
Table 1: Descriptive Statistics of Healthy Michigan Plan Managed Care Population, by disenrollment status

\begin{tabular}{|c|c|c|}
\hline 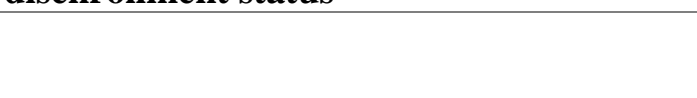 & $\begin{array}{l}\text { Disenroller } \\
(\mathrm{N}=39,289)\end{array}$ & $\begin{array}{l}\text { Continuously Enrolled } \\
\qquad(\mathrm{N}=156,206)\end{array}$ \\
\hline Female $(\%)$ & 51.1 & 63.1 \\
\hline Age (mean) & 37.6 & 40.4 \\
\hline First enrollment month (median) & Nov-14 & Oct-14 \\
\hline FPL percent & 85.0 & 76.4 \\
\hline \multicolumn{3}{|l|}{ Region } \\
\hline Northern Michigan(\%) & 9.9 & 10.4 \\
\hline Central Michigan(\%) & 30.9 & 31.1 \\
\hline Southern Michigan & 22.9 & 19.4 \\
\hline Detroit & 36.3 & 39.1 \\
\hline \multicolumn{3}{|l|}{ Race } \\
\hline White & 61.8 & 66.6 \\
\hline Black & 17.7 & 19.8 \\
\hline Other & 20.5 & 13.5 \\
\hline Monthly medical spending (mean \$) & 165.67 & 296.51 \\
\hline $\begin{array}{r}\text { Monthly number of chronic disease } \\
\text { claims (mean) }\end{array}$ & 0.24 & 0.42 \\
\hline Received premium statement (\%) & 24.5 & 20.1 \\
\hline Received copay statement (\%) & 27.4 & 40.4 \\
\hline Monthly Premium (mean \$) & 4.49 & 3.39 \\
\hline Monthly Copayment (mean \$) & 0.53 & 0.92 \\
\hline Received Healthy Behavior Reward & 14.9 & 25.3 \\
\hline
\end{tabular}

Notes: Population Inclusion Criteria: 1) Not part of special population (e.g. hospice, nursing home care, children's health programs) 2) Between 22 and 62 years of age 3) Enrolled in Healthy Michigan Plan Managed Care (HMPMC) before Sept 2015, so that we have at least 13 months of potential observation 4) At least 7 months of continuous HMP-MC enrollment 5) Income between 1\% and 133\% FPL

Disenroller: Drops HMP-MC after a spell of at least 7 months in the program up to 13 months in program.

Disenrollers must not come back to any Michigan Medicaid program for at least 6 months. Must have dropped from HMP-MC, i.e. not switched into another program and then dropped. Premium contribution and copayment means calculated by taking mean of $1 / 3$ of an enrollee's quarterly contribution or copayment invoices, net of any program reductions but not including past due amounts. 
Table 2. Main Estimates: Effect of Premiums on Medicaid Disenrollment

Effect of exceeding cutoff on

Treatment effect of

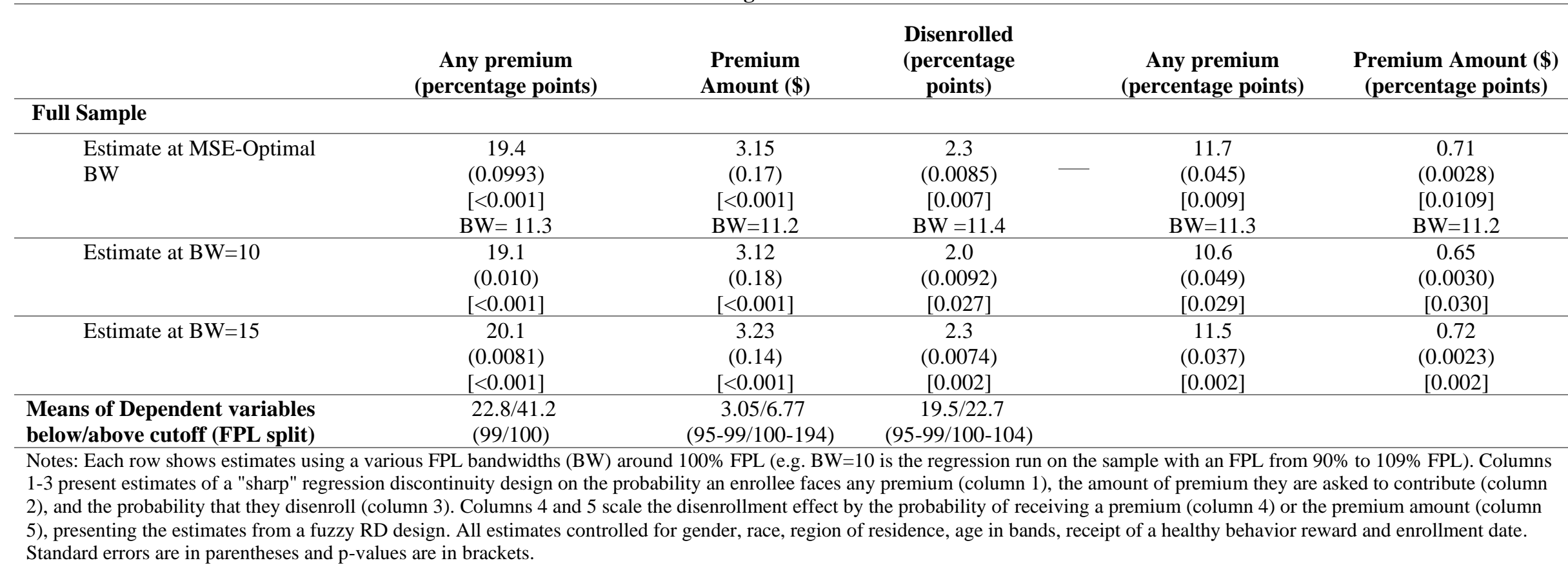




\begin{tabular}{|c|c|c|c|c|c|}
\hline & $\begin{array}{c}\text { Any premium } \\
\text { (percentage points) }\end{array}$ & $\begin{array}{c}\text { Premium } \\
\text { Amount (\$) }\end{array}$ & $\begin{array}{c}\text { Disenrolled } \\
\text { (percentage } \\
\text { points) }\end{array}$ & $\begin{array}{c}\text { Any premium } \\
\text { (percentage points) }\end{array}$ & $\begin{array}{c}\text { Premium Amount (\$) } \\
\text { (percentage points) }\end{array}$ \\
\hline \multicolumn{6}{|c|}{ Above Median Spending in first 7 months enrollment (>\$7/month) } \\
\hline Estimate at MSE-Optimal & 14.1 & 2.25 & -0.13 & 4.5 & 0.40 \\
\hline \multirow[t]{3}{*}{$\mathrm{BW}$} & $(0.014)$ & $(0.23)$ & $(0.014)$ & $(0.083)$ & $(0.0048)$ \\
\hline & {$[<0.001]$} & {$[<0.001]$} & {$[0.924]$} & {$[0.589]$} & {$[0.405]$} \\
\hline & $\mathrm{BW}=10.7$ & $\mathrm{BW}=12.8$ & $\mathrm{BW}=8.3$ & $\mathrm{BW}=10.7$ & $\mathrm{BW}=12.8$ \\
\hline \multirow[t]{3}{*}{ Estimate at $\mathrm{BW}=10$} & 13.9 & 2.22 & 0.49 & 3.5 & 0.22 \\
\hline & $(0.014)$ & $(0.26)$ & $(0.012)$ & $(0.088)$ & $(0.0055)$ \\
\hline & {$[<0.001]$} & {$[<0.001]$} & {$[0.690]$} & [0.691] & [0.6913] \\
\hline \multicolumn{6}{|c|}{ Below Median Spending in first 7 months enrollment $(<\$ 77 /$ month $)$} \\
\hline Estimate at MSE-Optimal & 23.8 & 3.87 & 3.6 & 13.4 & 0.82 \\
\hline \multirow[t]{3}{*}{ BW } & $(0.014)$ & $(0.25)$ & $(0.012)$ & $(0.058)$ & $(0.0036)$ \\
\hline & {$[<0.001]$} & {$[<0.001]$} & [0.002] & {$[0.0217]$} & {$[0.025]$} \\
\hline & $\mathrm{BW}=9.97$ & $\mathrm{BW}=9.6$ & $\mathrm{BW}=13.2$ & $\mathrm{BW}=9.97$ & $\mathrm{BW}=9.6$ \\
\hline \multirow[t]{3}{*}{ Estimate at $\mathrm{BW}=10$} & 23.8 & 3.90 & 3.2 & 13.4 & 0.82 \\
\hline & $(0.014)$ & $(0.24)$ & $(0.014)$ & $(0.058)$ & $(0.0035)$ \\
\hline & {$[<0.001]$} & {$[<0.001]$} & {$[0.018]$} & {$[0.020]$} & {$[0.021]$} \\
\hline \multicolumn{6}{|l|}{ Chronic Disease Claims } \\
\hline Estimate at MSE-Optimal & 14.8 & 2.47 & 1.3 & -3.1 & 0.53 \\
\hline \multirow[t]{3}{*}{ BW } & $(0.016)$ & $(0.021)$ & $(0.010)$ & $(0.10)$ & $(0.0041)$ \\
\hline & {$[<0.001]$} & {$[<0.001]$} & {$[0.191]$} & {$[0.750]$} & {$[0.199]$} \\
\hline & $\mathrm{BW}=7.6$ & $\mathrm{BW}=13.9$ & $\mathrm{BW}=14.2$ & $\mathrm{BW}=7.6$ & $\mathrm{BW}=13.9$ \\
\hline \multirow[t]{3}{*}{ Estimate at $\mathrm{BW}=10$} & 15.0 & 2.44 & 0.61 & 4.1 & 0.25 \\
\hline & $(0.014)$ & $(0.025)$ & $(0.012)$ & $(0.082)$ & $(0.0050)$ \\
\hline & {$[<0.001]$} & {$[<0.001]$} & {$[0.616]$} & [0.618] & {$[0.618]$} \\
\hline \multicolumn{6}{|l|}{ No Chronic Disease Claims } \\
\hline Estimate at MSE-Optimal & 22.8 & 3.69 & 3.3 & 13.6 & 0.84 \\
\hline \multirow[t]{3}{*}{ BW } & $(0.015)$ & $(0.27)$ & $(0.013)$ & $(0.067)$ & $(0.0042)$ \\
\hline & {$[<0.001]$} & {$[<0.001\}$} & {$[0.016]$} & {$[0.043]$} & {$[0.044]$} \\
\hline & $\mathrm{BW}=8.5$ & $\mathrm{BW}=8.5$ & $\mathrm{BW}=10.4$ & $\mathrm{BW}=8.5$ & $\mathrm{BW}=8.5$ \\
\hline \multirow[t]{3}{*}{ Estimate at $\mathrm{BW}=10$} & 23.3 & 3.77 & 3.3 & 14.2 & 0.88 \\
\hline & $(0.014)$ & $(0.25)$ & $(0.014)$ & $(0.060)$ & $(0.0037)$ \\
\hline & {$[<0.001]$} & {$[<0.001]$} & {$[0.016]$} & [0.018] & {$[0.019]$} \\
\hline
\end{tabular}


Notes: Each row shows estimates using a various FPL bandwidths (BW) around 100\% FPL (e.g. BW=10 is the regression run on the sample with an FPL from 90\% to 109\% FPL).

Columns 1-3 present estimates of a "sharp" regression discontinuity design on the probability an enrollee faces any premium (column 1), the amount of premium they are asked to

contribute (column 2), and the probability that they disenroll (column 3). Columns 4 and 5 scale the disenrollment effect by the probability of receiving a premium (column 4 ) or the premium amount (column 5), presenting the estimates from a fuzzy RD design. All estimates are controlled for gender, race, region of residence, age in bands, receipt of a healthy behavior reward and enrollment date. Standard errors are in parentheses and p-values are in brackets 


\section{Figure 1: Proportion of the Population with Premiums by Mean Federal Poverty Level}

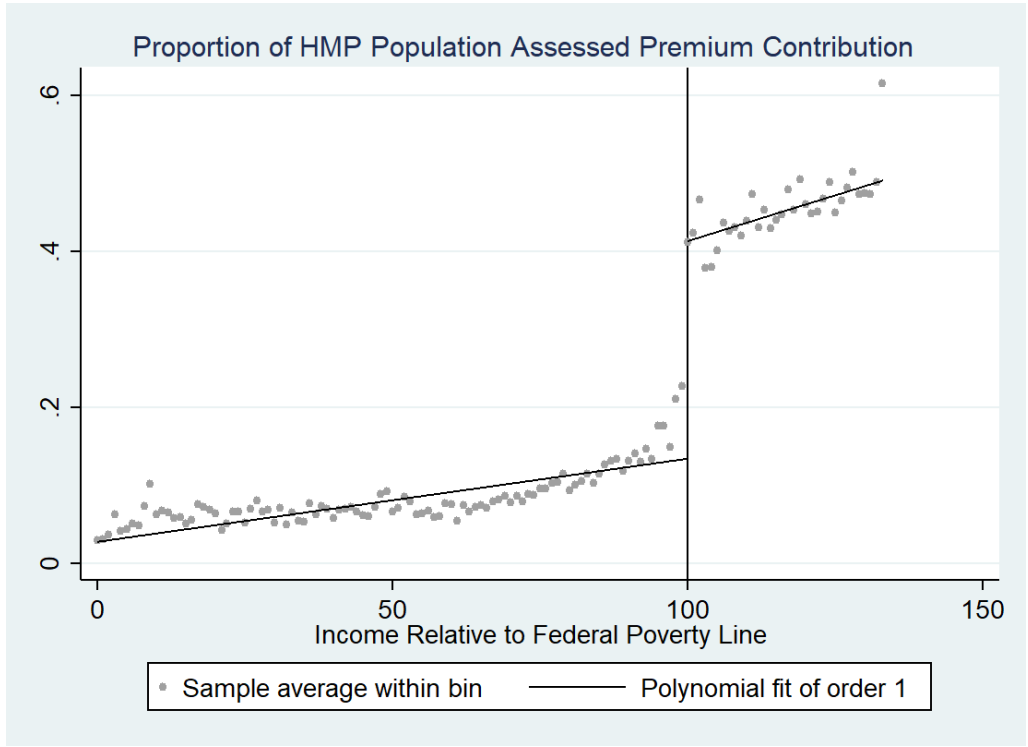

Notes: These data represent the share of enrollees with a premium at each income percentile relative to the federal poverty level, with a linear fit line on either side of the premium cutoff. The population represented here includes all enrollees in Michigan's Medicaid program with a positive income who were enrolled in a managed care plan for at least 7 months $(\mathrm{n}=195,495)$ 
Figure 2: Disenrollment by Mean Federal Poverty Level

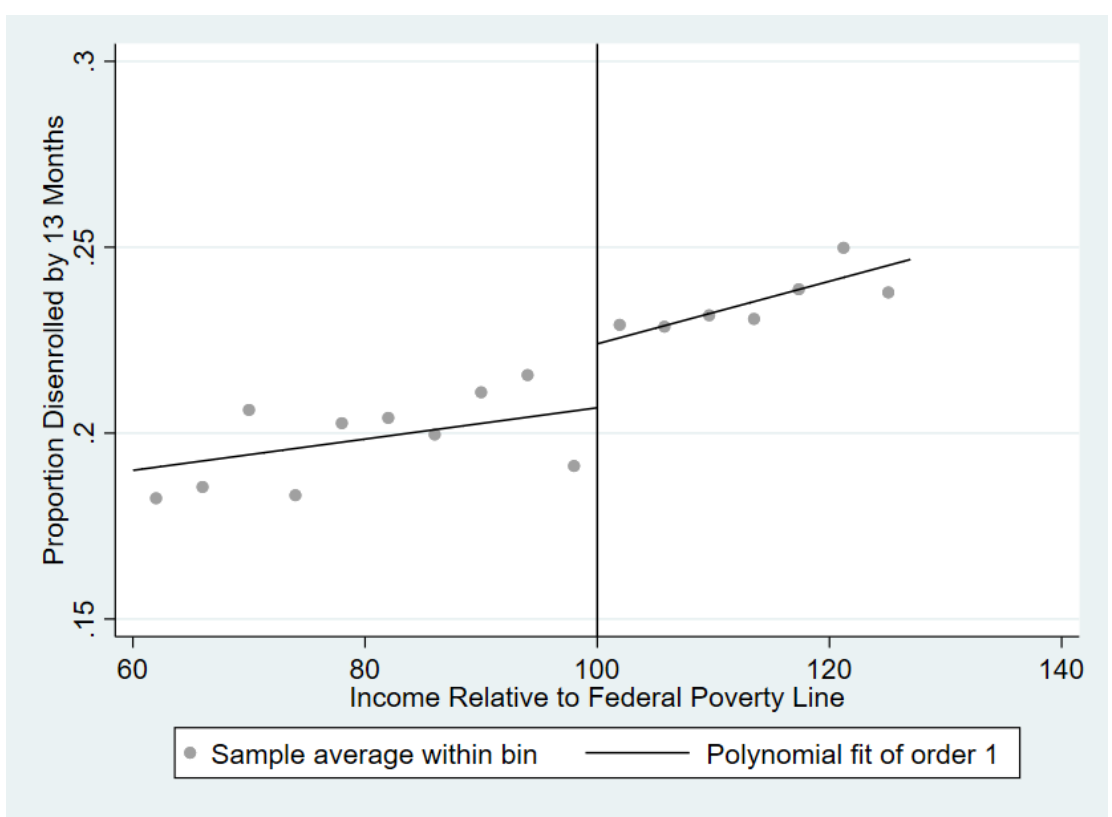

Notes: These data represent percentage of disenrollers at each federal poverty level, binned in groups of 4 to reduce noise, with a best-fitting line drawn through each scatterplot above and below the federal poverty line. The population represented here includes all enrollees in Michigan's Medicaid program with a positive income who were enrolled in a managed care plan for at least 7 months $(n=195,495)$. Data come from individual enrollment and premium files provided by Michigan DHHS. 
Figure 3: Disenrollment by Mean Federal Poverty Level split by 1) Below and Above Median Medical Spending and 2) Chronic Disease Diagnosis, both in first 7 months of HMP Managed Care Enrollment

Panel A: Below Median Medical Spending

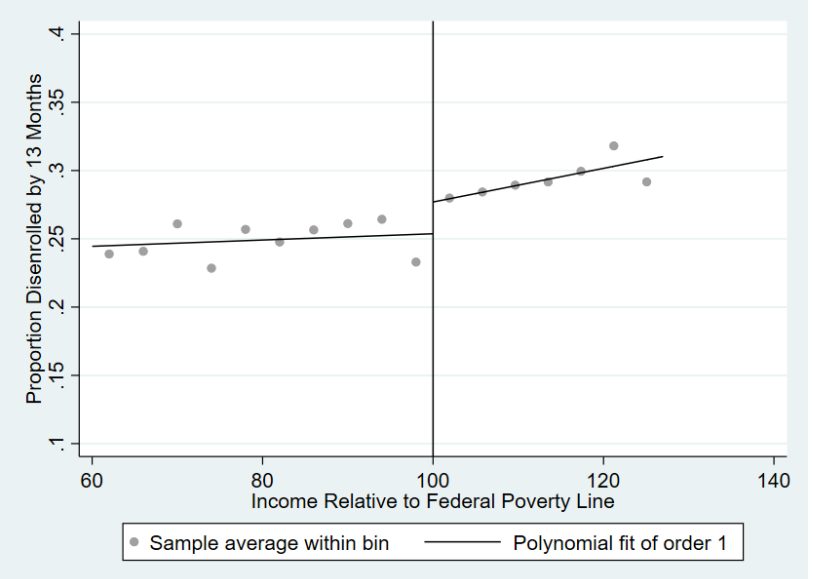

Panel C: No Chronic Disease Diagnosis

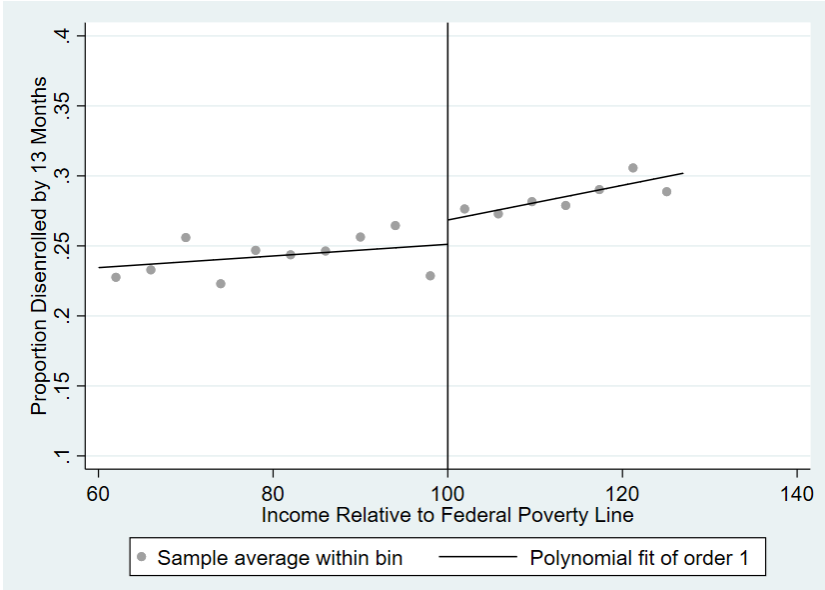

Panel B: Above Median Spending

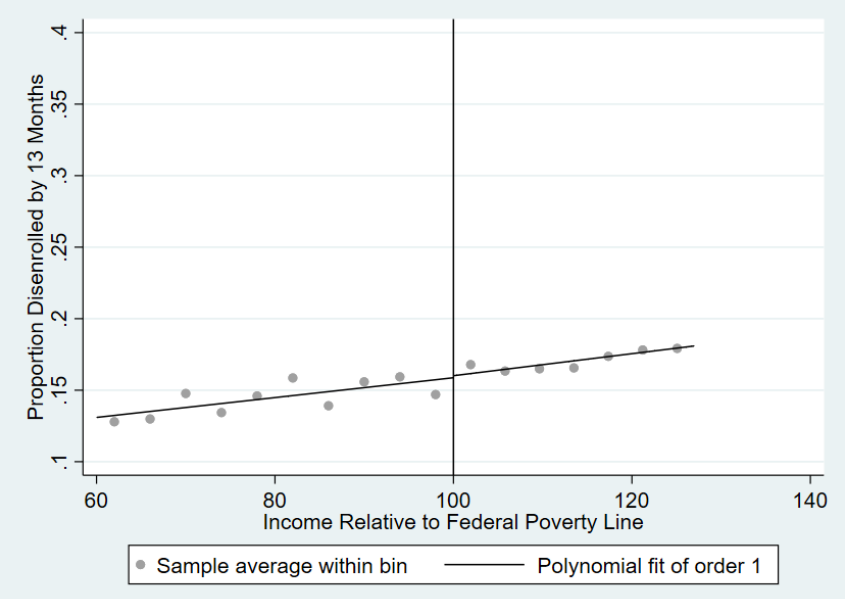

D. Chronic Disease Diagnosis

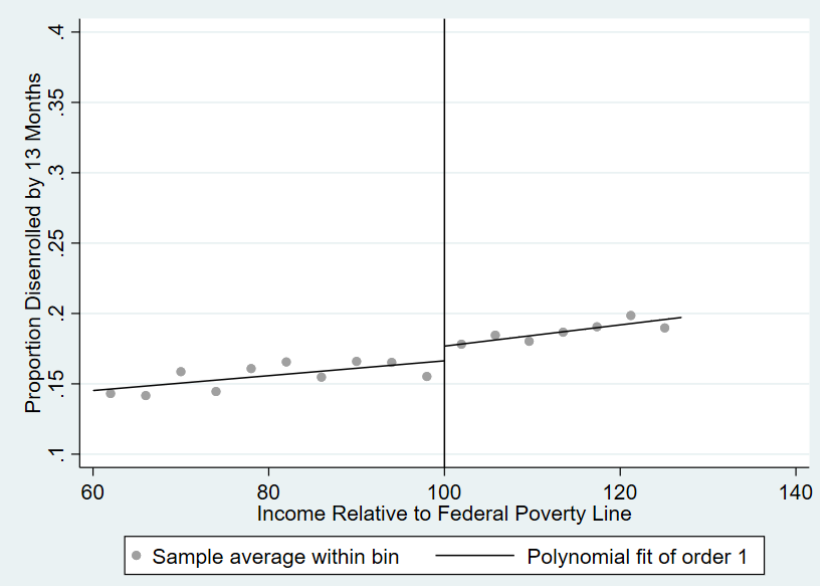

Notes: These data represent percentage of disenrollers each federal poverty level, at each federal poverty level, binned in groups of 4 to reduce noise with a bestfitting line drawn through each scatterplot, above and below the federal poverty line. The population represented here includes all enrollees in Michigan's

Medicaid program with a positive income who were enrolled in a managed care plan for at least 7 months $(n=195,495)$. The population is split by those who, at 
7 months, spent at or above the median amount (\$77) and those who spent below that amount. Data come from individual enrollment and cost-sharing files provided by Michigan DHHS. 